Reprod. Nutr. Dévelop., 1986, 26 (1 B), 325-326.

\title{
Création d'une fistule iléocaecale chez les équidés
}

H. BRUGËRE, G. CASTELLANI (*), Safia KAIDI, R. WOLTER

Ecole Nationale Vétérinaire,

94704 Maisons Alfort Cedex, France.

(*) Facultad de Ciencias Veterinarias de la U.N.A., Casilla de Correos N 1601 Asuncion, Paraguay.

Summary. A re-entrant cannulation of the intestine was performed in Equidae (ponies and donkeys) between the distal extremity of the ileum and the base of the caecum. Two donkeys were still alive ten months after the operation. This method can provide a convenient model for digestibility studies in Equidae.

La digestion chez les équidés comprend, en première analyse, des processus de digestion enzymatique se déroulant dans l'intestin grêle, et des processus de digestion microbienne résultant de l'activité des micro-organismes du gros intestin (caecum et côlon). Une des principales préoccupations des nutritionnistes est donc d'évaluer la part respective de ces deux étapes, c'est-à-dire, en fait, la contribution de la digestibilité précaecale à la digestibilité totale. Ceci implique de pouvoir mesurer le débit des digesta à la jonction iléocaecale, ainsi que d'y prélever les échantillons nécessaires aux déterminations biochimiques. Ces objectifs ont déjà été obtenus (Meyer, 1982) par la création d'une large fistule caecale donnant accès à l'iléon terminal et permettant la collecte des digesta. Notre travail a consisté à établir une fistule ré-entrante à la jonction iléocaecale, pour pouvoir recueillir la totalité de l'effluent iléal et le réintroduire, ensuite, dans le caecum. Ces essais ont été pratiqués chez six animaux de réforme, poneys et ânes, d'un poids compris entre 150 et $200 \mathrm{~kg}$. Les premiers animaux opérés ont permis une mise au point progressive du protocole opératoire dont la version définitive décrite ci-après a abouti, en fin de compte, à l'obtention de deux ânes équipés d'une fistule ré-entrante iléocaecale.

1. L'anesthésie comporte une prémédication par injection veineuse d'acépromazine $(0,66 \mathrm{mg} / \mathrm{kg})$. Trente minutes plus tard, l'injection d'un myorelaxant (éther glycérine de gaïacol, 80 à $110 \mathrm{mg} / \mathrm{kg}$ ) permet de coucher l'animal et d'éviter ses réactions de défense. L'anesthésie proprement dite est obtenue par le thiopental sodique injecté à la demande (dose moyenne de $7,5 \mathrm{mg} / \mathrm{kg}$ ).

2. Une laparotomie est pratiquée dans le creux du flanc droit selon une ligne parallèle aux fibres du muscle oblique interne (fig. 1).

3. L'accès à la cavité abdominale permettra l'interruption du transit digestif et la préparation des fistules.

3.1. Interruption du transit digestif normal : après avoir localisé la jonction iléocaecale, l'iléon est sectionné. Ceci peut être fait à la limite de la paroi caecale, ce qui correspond à la situation la plus souhaitable. Dans un cas, la longueur du mésentère nous a semblé insuffisante pour que l'abouchement de l'iléon à la peau puisse se faire sans traction excessive et sans risque de rupture vasculaire. La section a été pratiquée dans ce cas à $20-25 \mathrm{~cm}$ de la valvule iléocaecale. La section de l'iléon et la suture des deux extrémités est faite de préférence à l'aide d'une pince à agrafes (pince de Nakayama). Le moignon distal est enfoui par un surjet. 


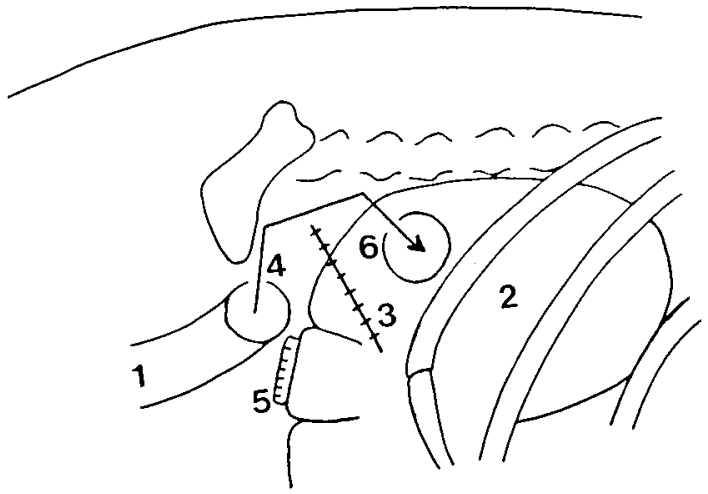

FIG. 1. - Schéma de principe du montage. (La flèche indique le sens du transit dans la canule réentrante, non représentée ici). 1 . Iléon ; 2. Caecum (base) ; 3. Incision principale ; 4 . Fistule iléale ; 5. Moignon iléal ; 6 . Fistule caecale.

3.2. Préparation des deux fistules: leur localisation est telle que l'iléon débouchera en dessous et un peu en arrière par rapport à l'incision principale, le caecum au-dessus et un peu en avant. Une pastille de peau est enlevée à chacun de ces emplacements, puis la paroi est « tunnellisé » pour permettre le passage de l'extrémité proximale de l'iléon et d'une portion de la base du caecum. Dans les deux cas, la paroi intestinale est fixée de façon provisoire à la peau.

4. La fermeture de l'incision principale est réalisée dès que possible, ce qui limite la contamination septique, le refroidissement de l'animal et la déshydratation par évaporation. Elle comporte trois plans, dont deux pour les muscles. Un drain est placé en région sous-cutanée.

5. Les fistules sont enfin achevées. Ceci nécessite tout d'abord de placer deux rangées de sutures reliant l'intestin à la paroi (l'une pour le plan musculopéritonéal, l'autre pour la peau). En second lieu, l'intestin doit être ouvert vers le milieu extérieur. Une bonne hémostase s'avère nécessaire, et elle est obtenue en plaçant des points en $U$ pour la rangée des sutures externes (peau + intestin). Les deux orifices sont ensuite équipés de canules en silastic reliées entre elles par un tube en épingle à cheveux.

6. Les soins post-opératoires ont consisté à administrer des réhydratants, des antibiotiques et une préparation analgésique et antispasmodique destinée à prévenir l'apparition de coliques. La réalimentation est progressive à partir du $2^{\mathrm{e}}$ ou $3^{e}$ jour.

Deux ânes opérés selon ce protocole ont présenté une période de survie compatible avec une utilisation expérimentale. Le premier a vécu dix mois, sa mort étant survenue à la suite d'une période où il recevait un régime peu appétible qui n'était consommé qu'en petite quantité ; l'autopsie n'a pas révélé de complication infectieuse. Le second est toujours vivant dix mois après l'opération.

Cette expérience montre qu'il est possible de réaliser une dérivation du contenu intestinal en région iléocaecale chez les équidés. Cette préparation fournit un modèle convenable pour l'étude de la digestibilité précaecale chez les équidés.

Meyer H. et al., 1982. Beiträge zur Verdauungsphysiologie des Pferdes. In Fortschritte in der Tierphysiologie und Tierernährung, Beih. Z. Tierphysiol. Tierernährg. Futtermittelkde, P. Parey Verlag, Berlin. 